\title{
US Perspectives in the Management of Psoriasis and Psoriatic Arthritis: Patient and Physician Results from the Population- Based Multinational Assessment of Psoriasis and Psoriatic Arthritis (MAPP) Survey
}

\author{
Mark G. Lebwohl ${ }^{1}$ - Arthur Kavanaugh ${ }^{2}$ - April W. Armstrong ${ }^{3}$ - Abby S. Van Voorhees ${ }^{4}$
}

Published online: 30 December 2015

(c) The Author(s) 2015. This article is published with open access at Springerlink.com

\begin{abstract}
Background The Multinational Assessment of Psoriasis and Psoriatic Arthritis (MAPP), a population-based survey of patients, dermatologists, and rheumatologists, was conducted for better understanding of the unmet needs of psoriasis and psoriatic arthritis (PsA) patients.

Objective To report results from US physicians and patients. Methods Adults were contacted by household telephone, using random digit dialing, and asked to participate if they had ever been diagnosed with psoriasis or PsA. Physicians were identified through national databases and contacted through random sampling methods.

Results In the USA, 1005 patients, 101 dermatologists, and 100 rheumatologists were surveyed. PsA had been diagnosed in 270 patients $(26.9 \%)$. Of those with psoriasis alone, fewer than $60 \%$ (versus $85.6 \%$ of PsA patients) had seen a healthcare provider within 12 months. Joint pain was reported by $51.8 \%$ of psoriasis patients without a diagnosis of PsA, and $37.6 \%$ of dermatologists cited their greatest challenge in managing PsA patients as being differentiating PsA from other arthritic diseases. Itching was reported by $36 \%$ of psoriasis patients versus $12 \%$ of dermatologists as the most
\end{abstract}

Mark G. Lebwohl

lebwohl@aol.com

1 The Icahn School of Medicine at Mount Sinai, Mount Sinai Medical Center, 5th Floor, 5 East 98th Street, Box 1048, New York, NY 10029, USA

2 School of Medicine, University of California, San Diego, La Jolla, CA, USA

3 Department of Dermatology, University of Southern California, Los Angeles, CA, USA

4 Department of Dermatology, Eastern Virginia Medical School, Norfolk, VA, USA important factor contributing to disease severity. Patients reported lower rates of current treatment than did dermatologists and rheumatologists. Conventional oral and biologic therapies were used by 24.9 and $17.7 \%$ of patients, respectively. Among patients who had received injectable biologics, treatment dissatisfaction was related to long-term safety/tolerability, injection-related anxiety/fear, and cost.

Conclusion This large, population-based survey identified unmet needs in the management of psoriasis and PsA patients in the USA, including assessment of disease severity, PsA diagnosis, undertreatment, and satisfaction with therapy.

\section{Key Points}

Both psoriasis and psoriatic arthritis remain undertreated in patients with moderate to severe disease.

Unmet needs exist in screening, assessing, diagnosing, and treating psoriasis patients who have symptoms of psoriatic arthritis.

Widespread dissatisfaction with current treatment options is reflected in patients not seeing a physician or not initiating or continuing therapies because of concerns with long-term safety, administration challenges, and cost.

Our findings underscore differences in perceptions between patients and physicians regarding disease severity and its impact on treatment selection, and highlight the need for ongoing communication between patients and physicians for better understanding of perceptions of disease severity, as well as treatment options and goals. 


\section{Introduction}

Psoriasis affects more than 7 million adults in the USA [1]. While the prevalence of psoriasis has remained generally stable at approximately $3 \%$ over the past decade $[1,2]$, the incidence nearly doubled between 1970 and 2000 [3]. Patients with psoriasis report a significant impact on their quality of life, including mental and social function [4-6]. In addition, approximately $30 \%$ of psoriasis patients also develop psoriatic arthritis (PsA) [7-9], which can add to the impact on quality of life, affecting physical function and leading to productivity loss and disability $[4,5,10,11]$. Because of the chronic, inflammatory nature of psoriasis and PsA, long-term systemic therapy is often necessary to control the signs and symptoms, and to improve physical function and quality of life for patients [7, 12]. Recent reports have identified undertreatment and treatment dissatisfaction as significant barriers to optimal care of psoriasis and PsA patients [9, 13].

The population-based Multinational Assessment of Psoriasis and Psoriatic Arthritis (MAPP) survey of 3426 patients and 781 dermatologists and rheumatologists in North America and Europe was designed to evaluate the diagnosis and ongoing management of patients with psoriasis and PsA from the patient and physician perspectives, as well as evaluating unmet treatment needs. The large volumes of data collected from the study were too extensive to include in a single publication. Results from the global patient and physician MAPP surveys, including the US patient findings, have been published separately [1416]. In this report, we explore findings from all US participants, including patients, dermatologists, and rheumatologists, to further investigate similarities and differences in attitudes toward disease severity and treatment, and to identify opportunities to improve the care of patients with psoriasis and PsA.

\section{Methods}

Abt SRBI, Inc. (New York, NY, USA) conducted the MAPP survey between June and August 2012. The study methodology has been reported previously [14, 16]. Briefly, this systematic household telephone survey used random digit dialing to identify a probability sample. Household members aged 18 years or older could participate if they had ever been diagnosed with psoriasis or PsA. Patients did not have to be under the care of a healthcare provider (HCP) at the time of the survey.

Dermatologists and rheumatologists were identified through the American Medical Association/American Osteopathic Association national database and were contacted through random sampling methods. Dermatologists were screened to ensure that $50 \%$ or more of their practice was devoted to medical dermatology.

\section{Results}

\subsection{Study Population}

A total of 1005 patients with psoriasis or PsA were surveyed in the USA; of these patients, 270 (26.9\%) reported a diagnosis of PsA. The mean age of surveyed patients was 57 years, and $61.3 \%$ were female (Table 1). Approximately $75 \%$ of patients reported at least one comorbid condition, with the most commonly reported conditions being general arthritis, hypertension, depression, diabetes, and heart disease. The mean age at symptom onset was 34 years, and the mean age at the time of patients' psoriasis diagnosis was 37 years. Patients with PsA reported a mean

Table 1 Patient demographics and clinical characteristics for the US portion of the Multinational Assessment of Psoriasis and Psoriatic Arthritis (MAPP) survey [15]

\begin{tabular}{ll}
\hline Characteristic & $n=1005^{\mathrm{b}}$ \\
\hline Age (years) & \\
Mean age (median) & $57(57)$ \\
Range (minimum-maximum) & $75(18-93)$ \\
Females [ $n(\%)]$ & $616(61.3)$ \\
Diagnosis of PsA [ $n(\%)]^{\mathrm{a}}$ & $270(26.9)$ \\
Mean age at onset of psoriasis (years) & 34 \\
Mean age at diagnosis of psoriasis (years) & 37 \\
Mean age at diagnosis of PsA (years) & 44 \\
Comorbidities $[n(\%)]$ & \\
Arthritis & $485(48.3)$ \\
Hypertension & $430(42.8)$ \\
Depression & $247(24.6)$ \\
Diabetes & $183(18.2)$ \\
Heart disease & $126(12.5)$ \\
Cancer & $76(7.6)$ \\
Other & $69(6.9)$ \\
Ulcerative colitis & $26(2.6)$ \\
Liver disease & $20(2.0)$ \\
Crohn's disease & $14(1.4)$ \\
Uveitis & $4(0.4)$ \\
None & $247(24.6)$ \\
\hline
\end{tabular}

The copyright for this material is owned by the National Psoriasis Foundation, and this material is reprinted courtesy of the National Psoriasis Foundation

PsA psoriatic arthritis

${ }^{\text {a }}$ With or without a separate diagnosis of psoriasis

b The $n$ value reflects the total number of patients surveyed; the actual number of patients answering each question may vary 
interval of 12.4 years between onset of skin symptoms and onset of joint symptoms; the mean age at the time of patients' PsA diagnosis was 44 years.

In the US physician portion of the MAPP survey, 1716 dermatologists and 1235 rheumatologists were screened, and 101 dermatologists and 100 rheumatologists completed the survey (Table 2). Physicians reported an average of 18 years in practice, and $98 \%$ reported that their practice was office based.

More than half of the dermatologists reported that their patients were diagnosed with psoriasis by another physician, while $41.1 \%$ of rheumatologists reported that they had made the diagnosis of PsA themselves. Referrals of patients newly diagnosed with psoriasis or PsA came in large part from primary care physicians $(\approx 55 \%)$. The majority of rheumatologists $(73.2 \%)$ reported being solely responsible for prescribing decisions for PsA patients, compared with $28.9 \%$ of dermatologists (Table 2). Approximately one third of the dermatologists and rheumatologists indicated co-management of patients, most commonly with a rheumatologist primarily being responsible for prescribing decisions and a dermatologist also monitoring skin symptoms.

\subsection{Factors Affecting Disease Severity}

Findings from the MAPP survey suggested that psoriasis patients and dermatologists may assess disease severity differently (Fig. 1a). More than one third of patients reported that itching was the most important factor contributing to their disease severity, followed by the location and size of lesions $(21.8 \%)$. In contrast, more than three times as many dermatologists $(76.2 \%)$ considered the location or size of the skin lesions to be the most important factor contributing to disease severity in their patients with psoriasis, while only one tenth of dermatologists cited itching (11.9\%).

Although PsA patients' and physicians' assessments of factors affecting disease severity were more consistent than those of psoriasis patients and physicians, itching continued to be rated as less important by physicians than by PsA patients. Approximately half of the PsA patients reported joint pain and swelling as the most important factors in disease severity (Fig. 1b). This was followed by itching, the location and size of the skin lesions, and flaking. The majority of dermatologists and rheumatologists considered joint pain or swelling to be the most important factor contributing to severity in PsA patients; only $2 \%$ of dermatologists and $2 \%$ of rheumatologists rated itching as the most important factor contributing to disease severity.
Table 2 Physician demographics and practice characteristics for the US portion of the Multinational Assessment of Psoriasis and Psoriatic Arthritis (MAPP) survey

\begin{tabular}{|c|c|c|}
\hline Characteristic & $\begin{array}{l}\text { Dermatologists, } \\
n=101\end{array}$ & $\begin{array}{l}\text { Rheumatologists, } \\
n=100\end{array}$ \\
\hline Number screened & 1716 & 1235 \\
\hline Mean time in practice (years) & 18.2 & 18.5 \\
\hline \multicolumn{3}{|l|}{ Practice setting $(\%)$} \\
\hline Urban & 29.7 & 42.0 \\
\hline Suburban & 60.4 & 55.0 \\
\hline Rural & 9.9 & 3.0 \\
\hline Office based practice $(\%)$ & 98.0 & 98.0 \\
\hline Hospital based practice $(\%)$ & 2.0 & 2.0 \\
\hline Multispecialty practice $(\%)$ & 12.9 & 41.0 \\
\hline $\begin{array}{l}\text { Mean number of } \\
\text { dermatologists in practice }\end{array}$ & 3.8 & 1.8 \\
\hline $\begin{array}{l}\text { Mean number of } \\
\text { rheumatologists in practice }\end{array}$ & 0.3 & 3.5 \\
\hline $\begin{array}{l}\text { Mean weekly number of } \\
\text { patients seen }\end{array}$ & 161.1 & 114.1 \\
\hline $\begin{array}{l}\text { Mean proportion of all visits } \\
\text { related to medical } \\
\text { dermatology }(\%)\end{array}$ & 82.0 & - \\
\hline $\begin{array}{l}\text { Mean proportion of all visits } \\
\text { related to psoriasis }(\%)\end{array}$ & 12.9 & - \\
\hline $\begin{array}{l}\text { Mean proportion of all visits } \\
\text { related to PsA }(\%)\end{array}$ & 3.4 & 15.5 \\
\hline \multicolumn{3}{|c|}{$\begin{array}{l}\text { Diagnosis of psoriasis (dermatologist) or skin symptoms in PsA } \\
\text { patients (rheumatologist) made by }\end{array}$} \\
\hline Other physician & 50.3 & 16.4 \\
\hline Self & 33.2 & 41.1 \\
\hline Other dermatologist & 6.5 & 39.5 \\
\hline \multicolumn{3}{|c|}{ Referrals of newly diagnosed psoriasis patients by } \\
\hline Primary care physician & 56.3 & - \\
\hline Other dermatologist & 5.3 & - \\
\hline Other specialist & 14.4 & - \\
\hline \multicolumn{3}{|c|}{ Referrals of newly diagnosed PsA patients by: } \\
\hline Primary care physician & 49.4 & 54.7 \\
\hline Other dermatologist & 4.0 & 27.6 \\
\hline Other specialist & 14.1 & 13.0 \\
\hline $\begin{array}{l}\text { Solely responsible for } \\
\text { prescribing decisions for PsA } \\
\text { patients }\end{array}$ & 28.9 & 73.2 \\
\hline $\begin{array}{l}\text { Rheumatologist responsible for } \\
\text { prescribing decisions; } \\
\text { dermatologist monitors skin } \\
\text { symptoms }\end{array}$ & 37.7 & 32.2 \\
\hline $\begin{array}{l}\text { Dermatologist responsible for } \\
\text { prescribing decisions; } \\
\text { rheumatologist monitors joint } \\
\text { symptoms }\end{array}$ & 31.6 & 13.1 \\
\hline
\end{tabular}

PsA psoriatic arthritis 
Fig. 1 Top five most important factors contributing to disease severity in psoriasis (a) and psoriatic arthritis (b), as reported by patients and physicians

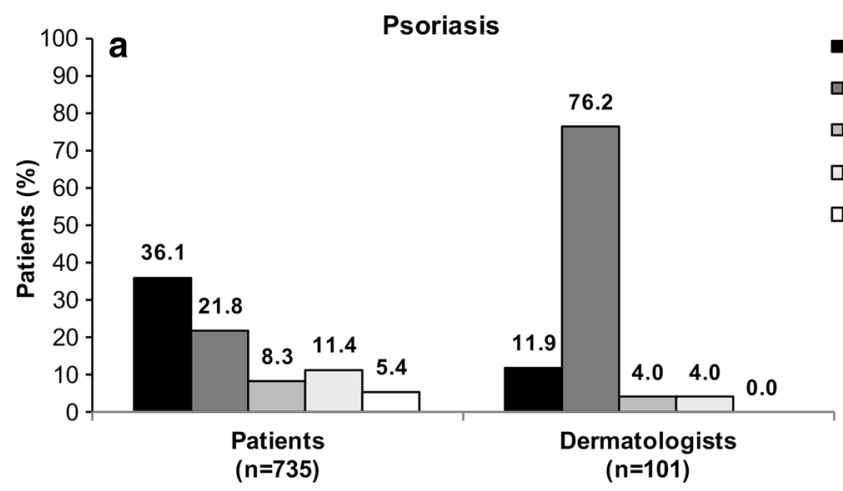

Psoriatic arthritis

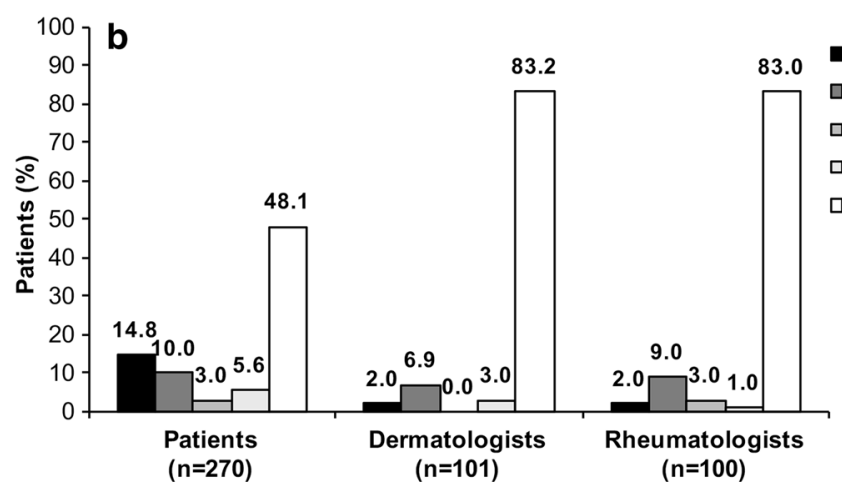

These observations are consistent with the global MAPP populations $[14,16]$ and highlight the differences between patients and physicians in the perception of disease severity and the factors that affect this perception.

\subsection{Assessment of Joint Pain}

Of the psoriasis patients who did not have a diagnosis of PsA, $51.8 \%$ reported joint pain; of these, $48.1 \%$ reported having more than four affected joints. In contrast, dermatologists reported a substantially lower percentage $(18.6 \%)$ of their psoriasis patients complaining of joint symptoms. This discrepancy may be due to variations in assessing joint pain (which is also associated with many inflammatory conditions beyond PsA) among patients. However, while most dermatologists reported discussing the possibility of developing joint disease with all $(57.4 \%)$ or some $(33.7 \%)$ of their psoriasis patients, $87.1 \%$ of dermatologists and $85.0 \%$ of rheumatologists acknowledged that PsA is likely underdiagnosed because of failure to connect skin and joint symptoms. In addition, $37.6 \%$ of dermatologists most often cited differentiating PsA from other arthritic diseases as the greatest challenge in managing PsA patients, while $25.0 \%$ of rheumatologists cited delayed referral as one of their greatest challenges.

\subsection{Physician Attitudes: Challenges in Patient Management}

Both dermatologists and rheumatologists reported that the management of patients with psoriasis and PsA can be challenging. According to $68.3 \%$ of the dermatologists, their psoriasis patients require more time and support than patients with other medical concerns, with $24.8 \%$ reporting management of psoriasis patients as complicated and $34.7 \%$ reporting it as time consuming. In addition, $32.7 \%$ of dermatologists reported that managing patients with PsA was complicated, while $26.7 \%$ of dermatologists said that managing these patients was time consuming. However, only $6.9 \%$ of dermatologists thought they would need to refer to or involve other specialists in the care of their PsA patients (and only $1 \%$ thought they would need to involve other specialists in the care of their psoriasis patients). Of the rheumatologists surveyed, $23 \%$ reported that their PsA patients require more time and support than their other patients.

In addition to the challenges of differentiating PsA from other arthritic conditions, dermatologists also cited challenges with the therapies for psoriasis and PsA, such as affordability (28.7-57.4\%), lack of long-term safety $(8.9-16.8 \%)$, and lack of effectiveness (12.9-14.9\%). Similarly, in addition to delayed referral, rheumatologists most often cited affordability (39\%), lack of effectiveness 
Table 3 Previous and current medical care in patients with psoriasis and psoriatic arthritis (PsA)

\begin{tabular}{lrl}
\hline Previous/current medical care & Psoriasis, $n=735(\%)$ & PsA, $n=270(\%)$ \\
\hline Seen an HCP in the previous 12 months & 57.4 & 85.6 \\
Type of HCP seen most often for psoriasis and PsA & & 22.2 \\
Dermatologist & 56.9 & 38.1 \\
Rheumatologist & 1.5 & 28.1 \\
General medicine or primary care physician & 30.2 & 10.0 \\
Other/unknown & 5.1 & 14.4 \\
Not seen an HCP in the previous 12 months & 42.6 & 68.0 \\
Ever seen a dermatologist (for psoriasis) or rheumatologist (for PsA) & 80.0 & 32.4 \\
Reasons for not seeing an HCP & & 20.6 \\
Symptoms were not bad enough & 34.1 & 23.5 \\
No symptoms & 19.9 & 2.9 \\
Did not think an HCP could help & 18.9 & 8.8 \\
Current treatment working & 10.6 & 2.9 \\
Cost or lack of insurance & 7.0 & 1.0 \\
Unable to get appointment & & \\
\hline
\end{tabular}

$H C P$ healthcare provider

(19\%), and lack of long-term safety (9\%) of current medications as the greatest challenges in treating PsA patients.

\subsection{Medical Care and Treatment Patterns}

\subsubsection{Patient Use of Medical Care}

Among the psoriasis patients, $42.6 \%$ reported that they had not seen an HCP in the previous 12 months. Of the $57.4 \%$ of psoriasis patients who had seen an HCP in the previous 12 months, $56.9 \%$ had seen a dermatologist and $30.2 \%$ had seen a primary care physician (Table 3 ). The most common reasons that psoriasis patients cited for not seeing an HCP were that the symptoms were not bad enough, there were no symptoms, they did not think an HCP could help, the current treatment was working, or they were unable to get an appointment (Table 3).

In contrast, the majority of PsA patients $(85.6 \%)$ had seen an HCP in the previous 12 months; these patients reported most often seeing a rheumatologist for their PsA, followed by a primary care physician and a dermatologist (Table 3). The reasons cited by PsA patients for not seeing an HCP in the previous year generally were similar to those cited by psoriasis patients, including that their symptoms were not bad enough, they did not think an HCP could help, there were no symptoms, the current treatment was working, and the cost or lack of insurance (Table 3).

\subsubsection{Current Treatments}

Overall, patients reported lower rates of current treatment than dermatologists and rheumatologists, with substantial differences noted in use of biologic therapy by psoriasis and PsA patients and use of conventional oral therapy by PsA patients (Tables 4, 5). Fewer than $10 \%$ of psoriasis patients reported use of conventional oral therapy or biologic therapy, compared with approximately $25 \%$ of PsA patients reporting use of each treatment. While $50 \%$ of PsA patients reported use of systemic therapy, half reported no treatment or reported use of topical medications only, leaving their joint disease untreated.

According to the physicians surveyed, use of conventional oral and biologic therapies was much higher than that reported by patients. Dermatologists reported use of conventional oral therapy in $12.7 \%$ of their psoriasis patients and $22.9 \%$ of their PsA patients, while rheumatologists reported use in $57.1 \%$ of PsA patients. Dermatologists reported use of biologic therapy in $35.8 \%$ of psoriasis patients and $46.9 \%$ of PsA patients, while rheumatologists reported use of biologic therapy in $52.7 \%$ of PsA patients. Some of these discrepancies in reported treatment rates probably arose because patients were not required to be seeing an $\mathrm{HCP}$ or receiving treatment to participate in the MAPP survey. However, even the proportion of physician-reported treatment indicates a level of undertreatment.

\subsection{Treatment Challenges and Burdens}

\subsubsection{Conventional Oral Therapy}

Of the 250 respondents who had ever used conventional oral therapies, about half found them burdensome. The reasons for the treatment burden most often cited by 
Table 4 Current treatment utilization in patients with moderate to severe psoriasis

\begin{tabular}{lcl}
\hline Therapy & Reported by psoriasis patients, $n=1005(\%)^{\mathrm{a}}$ & Reported by dermatologists, $n=101(\%)^{\mathrm{b}}$ \\
\hline Topical therapy & 40.0 & 79.1 \\
Conventional oral therapy & 5.8 & 12.7 \\
Biologic therapy & 7.9 & 35.8 \\
\hline
\end{tabular}

${ }^{a}$ Patients were asked to self-rate the severity of their disease on a 1-10 scale (1-3 for mild, 4-7 for moderate, 8-10 for severe)

b Treatment utilization is reported for patients with moderate to severe psoriasis, as estimated by dermatologists

Table 5 Current treatment utilization in patients with psoriatic arthritis (PsA)

\begin{tabular}{llll}
\hline Therapy & $\begin{array}{l}\text { Reported by PsA patients, } \\
n=270(\%)\end{array}$ & $\begin{array}{l}\text { Reported by dermatologists, } \\
n=101(\%)^{\mathrm{a}}\end{array}$ & $\begin{array}{l}\text { Reported by rheumatologists, } \\
n=100(\%)^{\mathrm{a}}\end{array}$ \\
\hline $\begin{array}{l}\text { Topical therapy } \\
\begin{array}{l}\text { Conventional oral } \\
\text { therapy }\end{array}\end{array}$ & 53.0 & 56.8 & 52.9 \\
$\begin{array}{l}\text { Biologic therapy } \\
\text { P }\end{array}$ & 24.1 & 22.9 & 57.1 \\
\end{tabular}

${ }^{a}$ Treatment utilization is reported for patients with moderate to severe PsA, as estimated by dermatologists or rheumatologists

patients were adverse effects and the need for laboratory monitoring (Fig. 2a). Patients most commonly discontinued conventional oral therapy because of safety and tolerability issues, or lack or loss of effectiveness (Fig. 3a). Similarly, the main reasons for dermatologists and rheumatologists not initiating or maintaining conventional oral therapy in psoriasis and PsA patients were related to long-term safety and tolerability concerns (Fig. 3b, c). In addition, one quarter of rheumatologists also cited lack of effectiveness as the main reason for not initiating conventional oral therapy for their PsA patients. Of the physicians who did not prescribe conventional oral therapy, because of patient refusal or concerns, the most common patient concerns were related to long-term safety, tolerability, and lifestyle modifications.
Fig. 2 Most common patientreported reasons for conventional oral therapy (a) and biologic therapy (b) being burdensome
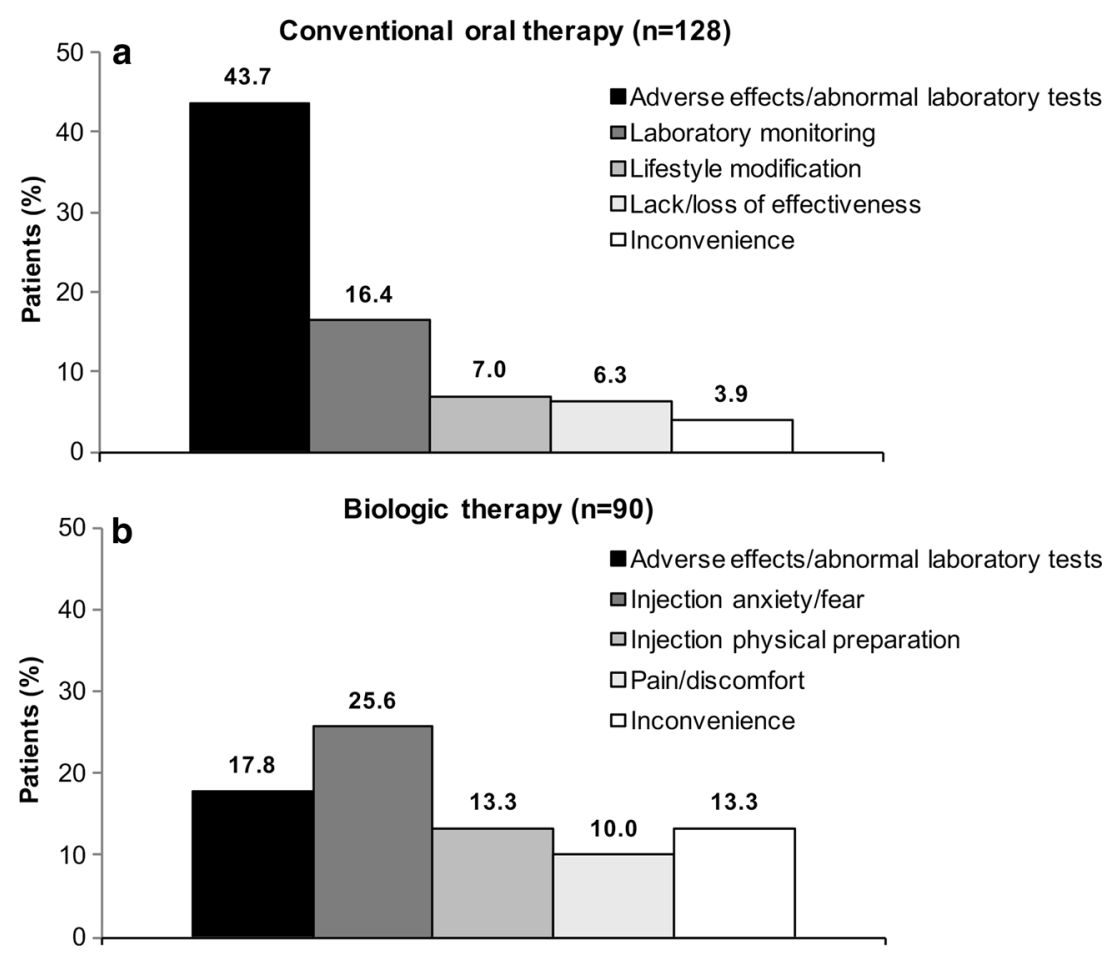


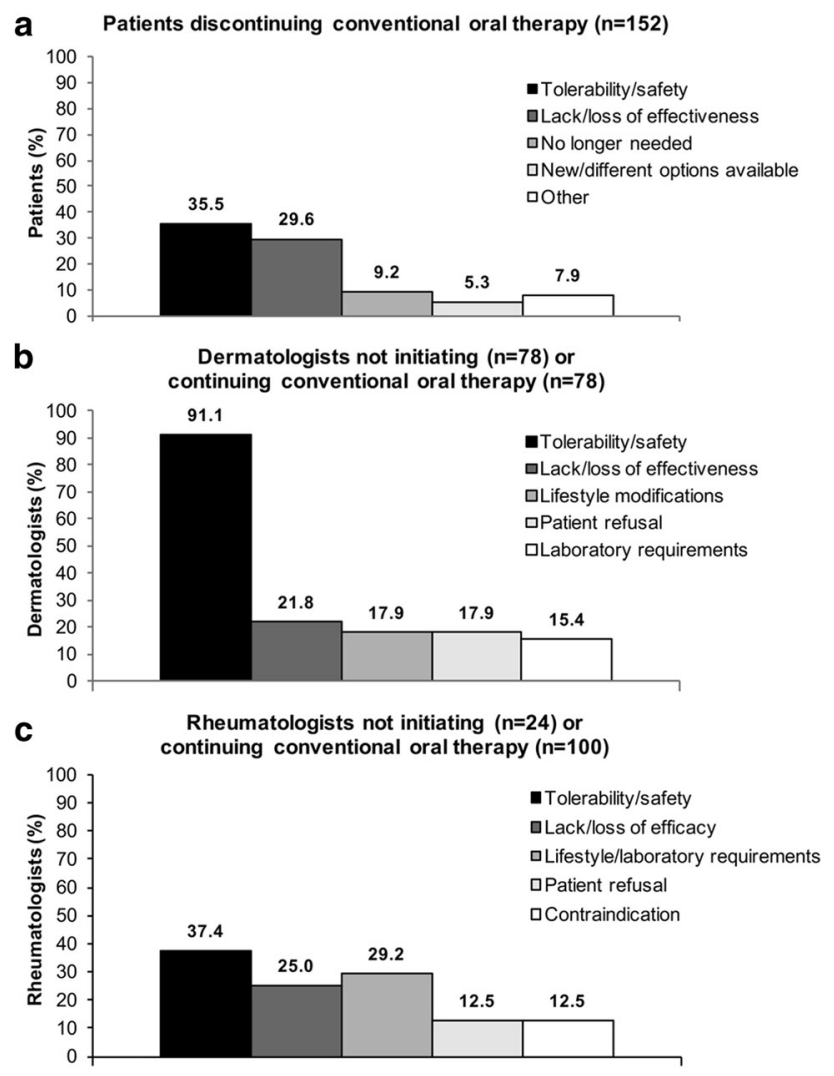

Fig. 3 Most common reasons for patients discontinuing conventional oral therapy (a) and for dermatologists (b) and rheumatologists (c) not initiating or continuing conventional oral therapy

\subsubsection{Biologic Therapy}

Among 90 patients who were using injectable biologics at the time of the survey, anxiety and fear related to injections and side effects were the most common reasons that $27 \%$ cited for finding them burdensome (Fig. 2b). As with conventional oral therapy, patients most commonly discontinued biologic therapy because of safety and tolerability concerns, and lack or loss of effectiveness; however, $13.7 \%$ of patients also reported cost as a reason for discontinuing biologic therapy (Fig. 4a).

Broad use of biologic agents was reported by three quarters of rheumatologists versus one third of dermatologists, yet $82.2 \%$ of dermatologists (versus $99.0 \%$ of rheumatologists) said they would initiate and manage biologic therapy themselves if their patient were a candidate. The main reasons that dermatologists and rheumatologists cited for not initiating or continuing biologic therapy were related to long-term safety and tolerability issues, as well as cost (Fig. 4b, c).

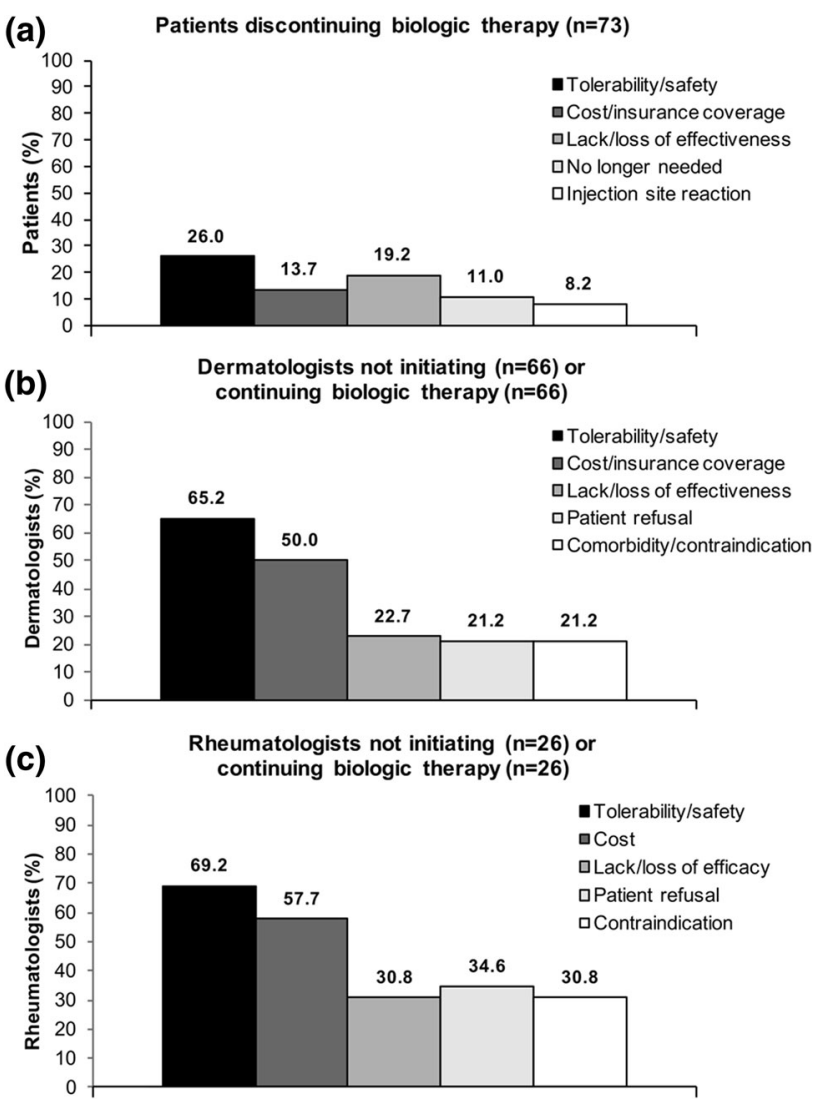

Fig. 4 Most common reasons for patients discontinuing biologic therapy (a) and for dermatologists (b) and rheumatologists (c) not initiating or continuing biologic therapy

\subsection{Satisfaction with Current Treatment Options}

The majority of psoriasis and PsA patients were somewhat satisfied or very satisfied with the effectiveness of conventional oral therapy (56.9 and $69.5 \%$, respectively) or biologic therapy (77.7 and $83.5 \%$, respectively), with more PsA patients than psoriasis patients reporting satisfaction. However, $33.9 \%$ of psoriasis patients and $39.7 \%$ of PsA patients reported being dissatisfied with the longterm safety of conventional oral therapy. Dissatisfaction with the long-term safety of biologic therapy was reported by $31.8 \%$ of psoriasis patients and $25.2 \%$ of PsA patients. Overall, $31.3 \%$ of psoriasis patients and $40.7 \%$ of PsA patients reported that their primary goals of therapy, including keeping symptoms at bay, reducing itching, and decreasing flaking, were not met with their current treatment.

In general, more physicians than patients reported satisfaction with treatment effectiveness. Among dermatologists, $76.3 \%$ reported satisfaction with conventional oral therapy effectiveness and $99.0 \%$ reported satisfaction with 
biologic therapy effectiveness, while 69.0 and $97.0 \%$ of rheumatologists, respectively, reported satisfaction. However, $53.2 \%$ of dermatologists and $17.0 \%$ of rheumatologists were dissatisfied with the long-term safety of conventional oral therapies; $17.8 \%$ of dermatologists and $15.0 \%$ of rheumatologists reported dissatisfaction with the long-term safety of biologic agents. Nearly $80 \%$ of dermatologists and more than $50 \%$ of rheumatologists reported that they were somewhat or very concerned about the overall long-term use of conventional oral or biologic therapy.

Overall, most survey respondents $(87.8 \%$ of patients, $98.0 \%$ of dermatologists, and $98.0 \%$ of rheumatologists) felt there was a strong or moderate need for better therapies. Among patients, $51.5 \%$ indicated that current therapies can be worse than the condition itself, compared with $30.7 \%$ of dermatologists and $12.0 \%$ of rheumatologists. In addition, $48.5 \%$ of dermatologists and $31.0 \%$ of rheumatologists reported that an important issue is patients leaving their practice because of frustration or dissatisfaction with currently available therapies.

\section{Discussion}

MAPP was unique in its design as a large-scale, multinational, population-based survey that included patients with psoriasis and/or PsA, as well as physicians. The results of the current report reflect the findings from the US respondents, including patients, dermatologists, and rheumatologists, with a goal of identifying the similarities and discrepancies in patient and physician responses, as well as the challenges and unmet needs of diagnosis, assessment, and treatment.

To participate in the MAPP survey, patients were not required to be under the care of a physician, receiving treatment, or a member of a patient organization. A substantial proportion of patients who participated had not seen an HCP for their psoriasis within the previous 12 months. The reported reasons included that they did not feel an HCP could help, demonstrating frustration with the current management options. This was mirrored by approximately half of the dermatologists and one third of the rheumatologists, who agreed that they were concerned about patients leaving their practice because of frustration or dissatisfaction with currently available therapies. Of those patients who had seen an HCP within the previous 12 months, approximately $30 \%$ of both psoriasis and PsA patients saw a primary care physician, not a specialist, which is slightly higher than the rate of $22 \%$ recently reported by the National Psoriasis Foundation (NPF) [17].

When asked about factors that affect disease severity, patient and physician responses differed. Patients, especially those with psoriasis, consistently placed more emphasis on pruritus than did physicians. US psoriasis patients [15] and global psoriasis patients [14] reported itching as the most important factor contributing to disease severity, followed by the location and size of lesions. In contrast, just over $10 \%$ of US dermatologists cited itching as the most important factor. This finding may be related to the fact that itching is not currently captured in most physician assessments or in the most commonly used psoriasis assessment tools, including those used in clinical trials but not often in practice, such as the Psoriasis Area and Severity Index and the physician's global assessment of disease severity [18]. Even among PsA patients, itching was cited by nearly $15 \%$ of patients as the most important factor contributing to disease severity. Overall, these findings suggest that patients may assess disease severity differently from physicians, and tend to consider symptoms beyond those usually assessed in a physician visit. Improved communication between patients and physicians may help to foster the understanding of all aspects of disease that impact patient quality of life.

Joint pain was reported by more than half of the patients with a diagnosis of psoriasis alone. However, fewer than $20 \%$ of dermatologists reported that their psoriasis patients had joint pain. Although more than three quarters of both dermatologists and rheumatologists agreed that PsA may go undiagnosed because of failure to connect skin and joint symptoms, fewer than $60 \%$ of dermatologists discussed this risk with all of their psoriasis patients. A separate report recently highlighted the underdiagnosis of PsA in dermatology treatment centers, where approximately $40 \%$ of patients diagnosed with PsA by a rheumatologist had not been diagnosed by their dermatologist [9].

The MAPP survey included additional questions about managing PsA patients that may provide more insights into the potential underdiagnosis of this disease. More than one third of the surveyed US dermatologists reported that the greatest challenge in managing their PsA patients was differentiating PsA from other arthritic diseases. However, fewer than $10 \%$ felt they would need to refer to or involve other specialists in the care of their PsA patients. The surveyed US rheumatologists reported delayed referrals as the second most common challenge in treating PsA patients. Fewer than $40 \%$ of physicians reported co-management of PsA patients. Most often, this relationship consisted of a rheumatologist primarily being responsible for prescribing decisions and a dermatologist monitoring skin symptoms. A recent review and meta-analysis provided recommendations for dermatologists regarding screening, diagnosing, and treating PsA patients [19]. Educating dermatologists to routinely discuss and evaluate joint disease with their psoriasis patients-and providing guidance on when to refer them to a rheumatologist—may 
bring about more timely diagnosis and treatment of patients with PsA. Co-management and/or collaborative efforts among dermatologists and rheumatologists may ensure that psoriasis patients, while receiving care for their skin disease, are also adequately screened and further assessed for the presence of PsA.

Despite expanding therapeutic options for managing psoriasis and PsA patients, undertreatment remains a challenge $[9,13]$. Although approximately $70 \%$ rated their disease as moderate or severe [15], US patients participating in the MAPP survey reported low treatment rates, with only about $10 \%$ of psoriasis patients reporting treatment with conventional oral or biologic therapy. US dermatologists reported nearly three times greater current use of conventional oral therapies and more than seven times greater current use of biologic agents for their psoriasis patients than were reported by the surveyed US patients. Likewise, while dermatologists and rheumatologists reported systemic therapy use in the majority of PsA patients, only half of the PsA patients reported current use of oral or biologic therapy. There are several possible explanations for these discrepancies. Patients included in the MAPP survey were not required to be seeing an HCP or receiving treatment at the time of the survey, and patients and physicians were not matched. Patients may have been seeing another specialist or a primary care physician for their disease. It is possible that seeing a primary care physician rather than a physician specializing in dermatologic or rheumatic conditions contributed to the lower treatment rates reported by the patients than by the participating physicians. In addition, approximately $20 \%$ of psoriasis patients reported having no skin lesions as reasons for not seeing an HCP, suggesting that treatment would not be required. However, although higher rates of treatment were reported by physicians than by patients, physicians indicated that more than $30 \%$ of patients were still not receiving systemic therapy for their moderate to severe psoriasis or for PsA at the time of the survey, in contrast to guideline recommendations [20, 21]. In addition, other surveys have also shown a high level of undertreatment. In the 2011 NPF survey, up to one third of patients were not receiving treatment for their moderate or severe psoriasis, and of the patients who were receiving treatment, an estimated 20-30\% were receiving topical monotherapy [13].

The complexity and time-consuming nature of treating patients with psoriasis and PsA may contribute to undertreatment. Most dermatologists reported that managing their psoriasis patients takes up more of their time than managing other patients, and approximately one third of dermatologists and rheumatologists described the care of patients with PsA as complicated and time consuming. Comorbidities, which were reported by the majority of patients, may also be a contributing factor to the complexity of treating psoriasis and PsA patients. In addition, multiple physicians providing care to an individual patient may lead to miscommunication or confusion over roles in prescribing decisions, and may contribute to lower treatment rates, emphasizing the importance of communication among HCPs.

Dissatisfaction with current therapies may impact disease management and treatment. The most commonly cited barriers to use of conventional oral therapy, across patients, dermatologists, and rheumatologists, were concerns with long-term safety and tolerability, and lack of effectiveness. Patients also reported laboratory monitoring (16.4\%) and lifestyle modification (7.0\%) as being among the top three reasons contributing to the burden of these therapies. Opinions on the barriers to use of biologic therapy differed among patients and physicians. Patients reported anxiety about and preparation of injections as the most common reasons for therapy being burdensome, and concerns with safety and tolerability or lack of effectiveness as reasons for discontinuation, while dermatologists and rheumatologists cited lack of long-term safety and cost as the most common reasons for not initiating or continuing biologic therapy. The discrepancy in the importance of cost to patients and physicians may reflect the effort required to determine insurance eligibility and ensure reimbursement before prescribing, which falls heavily on physicians versus patients, thus cost may not be considered an issue by patients. The reasons for treatment discontinuation are generally consistent with those found in a report of 1095 patients with moderate to severe psoriasis treated in dermatology clinics within the USA [22]. A survey of NPF members also found a high rate of dissatisfaction with treatment among psoriasis patients (52.3\%) and PsA patients $(45.5 \%)$, and cited adverse effects and efficacy as the main reasons for patient discontinuation of biologic therapy, along with inadequate insurance coverage and cost [13].

Overall, the results of the MAPP survey among the US respondents were consistent with those of global respondents; however, some differences among physicians and patients were noted. For patients with moderate to severe psoriasis, a higher proportion of US dermatologists reported treating patients with biologic therapies, compared with dermatologists globally (35.8 versus $19.6 \%$, respectively) [16]. Likewise, higher proportions of US dermatologists and rheumatologists reported treating PsA patients with biologic therapies, compared with dermatologists and rheumatologists globally (46.9-52.7 versus 30.6-33.4\%) [16]. The challenges of managing psoriasis and PsA patients identified by US physicians and global physicians were similar; however, affordability of therapies was considered a treatment challenge for psoriasis and PsA by a greater proportion of US physicians than global physicians 
(28.7-57.4 \% for US physicians versus $22.0-36.1 \%$ for global physicians) [16]. Survey responses among US patients generally reflected those of the global population. Differences between US patient responses and global patient responses to the MAPP survey have been reviewed previously; these include a higher proportion of US patients than global patients reporting comorbidities such as arthritis (48.3 versus $34.4 \%)$ and hypertension (42.8 versus $32.7 \%$ ) [14, 15]. Treatment patterns reported by US and global patients were generally similar, as were the reasons identified for treatment discontinuation or for not initiating treatments.

The findings from the MAPP survey are limited by survey-related factors, which include lack of a control group and reliance on accurate recall and interpretation of questions. Because the survey was blinded, respondents could not be contacted again to follow up or clarify answers. Although patients and physician results are compared within this report, the patients and physicians were not matched, and the survey was not designed to directly compare specific disease or treatment differences.

\section{Conclusion}

The findings from the US MAPP survey population highlight the differences in perceptions between physicians and patients with psoriasis and/or PsA regarding disease severity and the impact on treatment choices. Patients with psoriasis consistently reported that itching, which is usually not captured in current assessment tools, is the most influential factor in determining psoriasis severity, whereas physicians reported the size or location of lesions as leading factors contributing to disease severity. Psoriasis patients should be screened and assessed routinely for symptoms of PsA, and dermatologists need tools to differentiate PsA from other rheumatic diseases. These enhancements, along with improving referral rates and collaboration between dermatologists and rheumatologists, may lead to improved treatment of psoriasis and PsA. Ongoing communication between patients and physicians is needed for better understanding of perceptions of disease severity, discussion and agreement on treatment options, and establishment of realistic treatment goals. The need for safe, effective, and easy-to-use therapies for both psoriasis and PsA remains.

\section{Compliance with Ethical Standards}

Mark G. Lebwohl is an employee of the Mount Sinai Medical Center, which receives research funds from Amgen, Anacor, Aqua, Canfite Biopharma, Celgene, Clinuvel, Coronado Biosciences, Eli Lilly, Ferndale, Janssen Biotech, LEO Pharma, Merz, Novartis, Pfizer, Sandoz, and Valeant. Arthur Kavanaugh has received grants (or has grants pending) for clinical research from AbbVie, Amgen, Celgene, Eli Lilly, Janssen, and Novartis. April W. Armstrong has received grants (or has grants pending) from AbbVie, Eli Lilly, and Janssen; and has received consulting fees or honoraria from AbbVie, Amgen, Celgene, Eli Lilly, Janssen, Merck, Novartis, and Pfizer. Abby S. Van Voorhees has received grants (or has grants pending) from AbbVie and Amgen; has received consulting fees or honoraria from Amgen, Aqua, AstraZeneca, Celgene, Dermira, Janssen, LEO Pharma, Novartis, and Pfizer; and has received support for travel to meetings for this study, this manuscript, or other purposes from Celgene.

Funding The MAPP survey was sponsored by Celgene Corporation. The authors received editorial support in the preparation of the manuscript from Peloton Advantage, LLC, Jennifer Schwinn, RPh, and Amy Zannikos, PharmD, funded by Celgene Corporation. However, the authors directed and are fully responsible for all content and editorial decisions for this manuscript.

Open Access This article is distributed under the terms of the Creative Commons Attribution-NonCommercial 4.0 International License (http://creativecommons.org/licenses/by-nc/4.0/), which permits any noncommercial use, distribution, and reproduction in any medium, provided you give appropriate credit to the original author(s) and the source, provide a link to the Creative Commons license, and indicate if changes were made.

\section{References}

1. Rachakonda TD, Schupp CW, Armstrong AW. Psoriasis prevalence among adults in the United States. J Am Acad Dermatol. 2014;70:512-6.

2. Kurd SK, Gelfand JM. The prevalence of previously diagnosed and undiagnosed psoriasis in US adults: results from NHANES 2003-2004. J Am Acad Dermatol. 2009;60:218-24.

3. Icen M, Crowson CS, McEvoy MT, Dann FJ, Gabriel SE, Maradit Kremers H. Trends in incidence of adult-onset psoriasis over three decades: a population-based study. J Am Acad Dermatol. 2009;60:394-401.

4. Strand V, Sharp V, Koenig AS, et al. Comparison of healthrelated quality of life in rheumatoid arthritis, psoriatic arthritis and psoriasis and effects of etanercept treatment. Ann Rheum Dis. 2012;71:1143-50.

5. Armstrong AW, Schupp C, Wu J, Bebo B. Quality of life and work productivity impairment among psoriasis patients: findings from the National Psoriasis Foundation survey data 2003-2011. PLoS One. 2012;7:e52935.

6. Darjani A, Heidarzadeh A, Golchai J, et al. Quality of life in psoriatic patients: a study using the Short Form-36. Int J Prev Med. 2014;5:1146-52.

7. Gladman DD, Antoni C, Mease P, Clegg DO, Nash P. Psoriatic arthritis: epidemiology, clinical features, course, and outcome. Ann Rheum Dis 2005;64(Suppl 2):ii14-ii17.

8. Boehncke WH, Menter A. Burden of disease: psoriasis and psoriatic arthritis. Am J Clin Dermatol. 2013;14:377-88.

9. Mease PJ, Gladman DD, Papp KA, et al. Prevalence of rheumatologist-diagnosed psoriatic arthritis in patients with psoriasis in European/North American dermatology clinics. J Am Acad Dermatol. 2013;69:729-35.

10. Zhu TY, Tam LS, Leung YY, et al. Socioeconomic burden of psoriatic arthritis in Hong Kong: direct and indirect costs and the influence of disease pattern. J Rheumatol. 2010;37:1214-20. 
11. Singh JA, Strand V. Spondyloarthritis is associated with poor function and physical health-related quality of life. J Rheumatol. 2009;36:1012-20.

12. American Academy of Dermatology and AAD Association. Position statement for maintenance therapy for psoriasis patients. American Academy of Dermatology and AAD Association. 2012. https://www.aad.org/Forms/Policies/Uploads/PS/PS-Maintenance \%20Therapy\%20for\%20Psoriasis\%20Patients.pdf. Accessed 3 Feb 2015.

13. Armstrong AW, Robertson AD, Wu J, Schupp C, Lebwohl MG. Undertreatment, treatment trends, and treatment dissatisfaction among patients with psoriasis and psoriatic arthritis in the United States: findings from the National Psoriasis Foundation surveys, 2003-2011. JAMA Dermatol. 2013;149:1180-5.

14. Lebwohl MG, Bachelez H, Barker J, et al. Patient perspectives in the management of psoriasis: results from the population-based Multinational Assessment of Psoriasis and Psoriatic Arthritis survey. J Am Acad Dermatol. 2014;70:871-81.

15. Lebwohl MG, Kavanaugh A, Armstrong AW, Van Voorhees AS, Kalb RE. Patient perspectives on psoriasis management: US results of the population-based Multinational Assessment of Psoriasis and Psoriatic Arthritis survey. Psoriasis Forum. 2014;20:124-31.

16. van de Kerkhof PCM, Reich K, Kavanaugh A, et al. Physician perspectives in the managment of psoriasis and psoriatic arthritis: results from the population-based Multinational Assessment of
Psoriasis and Psoriatic Arthritis survey. J Eur Acad Dermatol Venereol. 2015;29:2002-10.

17. Bhutani T, Wong JW, Bebo BF, Armstrong AW. Access to health care in patients with psoriasis and psoriatic arthritis: data from National Psoriasis Foundation survey panels. JAMA Dermatol. 2013;149:717-21.

18. Feldman SR, Krueger GG. Psoriasis assessment tools in clinical trials. Ann Rheum Dis 2005;64(Suppl 2):ii65-ii68.

19. Richard MA, Barnetche T, Rouzaud M, et al. Evidence-based recommendations on the role of dermatologists in the diagnosis and management of psoriatic arthritis: systematic review and expert opinion. J Eur Acad Dermatol Venereol. 2014;28(Suppl 5):3-12.

20. Menter A, Gottlieb A, Feldman SR, et al. Guidelines of care for the management of psoriasis and psoriatic arthritis: section 1 . Overview of psoriasis and guidelines of care for the treatment of psoriasis with biologics. J Am Acad Dermatol. 2008;58:826-50.

21. Gottlieb A, Korman NJ, Gordon KB, et al. Guidelines of care for the management of psoriasis and psoriatic arthritis: section 2 . Psoriatic arthritis: overview and guidelines of care for treatment with an emphasis on the biologics. J Am Acad Dermatol. 2008;58:851-64.

22. Yeung H, Wan J, Van Voorhees AS et al. Patient-reported reasons for the discontinuation of commonly used treatments for moderate to severe psoriasis. J Am Acad Dermatol. 2013;68:64-72. 